\title{
Presencia de exostosis orales en pacientes de la ciudad de Temuco, Chile
}

\section{Presence of oral exostoses on patients of Temuco, Chile}

\author{
Fuentes Fernández R*, Borie Echevarría E*, Sanhueza Campos A**, \\ Rebolledo Soto $\mathrm{K}^{* * *}$, Parra Villagran P***
}

\section{RESUMEN}

La palabra torus proviene del latín que significa tumor o protuberancia circular, el cual no es considerado una condición patológica. Se examinaron un total de 159 pacientes al azar en las clínicas odontológicas de la Universidad de La Frontera, Temuco para determinar la prevalencia de exostosis en la población. A estos pacientes se les aplicó una ficha clínica con el fin de relacionar esta condición con algunos posibles factores asociados. Se determinó una prevalencia de $5,66 \%$, con predominio en el género femenino, en el sector mandibular. Si bien algunos factores estudiados mostraron ciertas tendencias ninguno, las características clínicas del torus mostró resultados estadísticamente significativos.

Palabras clave: Torus, torus palatino, torus mandibular, exostosis, condición anatómica.

\section{SUMMARY}

The torus comes from Latin word that means circular prominence or tumor and which is not considered like a pathological condition. 159 patients were evaluated randomly in the dental clinics of Universidad de La Frontera, Temuco, to determine the prevalence of exostoses on this population. These patients filled a tab in order to relate this condition with some associated factors. A prevalence of $5.66 \%$ was observed with predominance in female gender, in mandibular zone. While certain factors studied showed no trends except for clinical features, none exhibited statistically significant results.

Key words: Tori, torus palatinus, torus mandibularis, exostoses, anatomical condition.

Fecha de recepción: 29 de abril de 2011.

Aceptado para publicación: 12 de mayo de 2011.

Fuentes Fernández R, Borie Echevarría E, Sanhueza Campos A, Rebolledo Soto K, Parra Villagran P. Presencia de exostosis orales en pacientes de la ciudad de Temuco, Chile. Av. Odontoestomatol 2012; 28 (2): 63-69.

* Departamento de Odontología Integral. Facultad de Medicina. Universidad de La Frontera, Temuco, Chile.

** Departamento de Matemáticas y Estadística. Facultad de Ingeniería, Ciencias y Administración. Universidad de La Frontera, Temuco, Chile.

*** Alumnos pregrado Odontología. Universidad de La Frontera, Temuco, Chile.

\section{INTRODUCCIÓN}

Las exostosis o torus son excrecencias óseas convexas y de superficie lisa, cuyo crecimiento es lento y progresivo. Están compuestas por una cortical ósea densa y escaso hueso esponjoso recubierto por una delgada capa de mucosa pobremente irrigada $(1,2,3)$. Pueden ser uni o bilaterales, bien definidos y generalmente asintomáticos (3). La palabra torus tiene su origen en el latín, que significa tumor o protuberancia 
circular (4) y no se considera como una condición patológica (5), sino una variación anatómica (6).

Los torus pueden clasificarse en dos grandes tipos según su localización, en torus palatino (TP) a lo largo de la sutura palatina mediana o a cada lado de ella $(7,8)$, y torus mandibular (TM) ubicado en el sector lingual sobre la línea milohioidea, pudiendo extenderse desde el canino hasta la zona de los primeros molares inferiores $(8,9,10)$.

Autores como Lee y cols. (1) clasifican los TP según su ubicación, en Tipo I (desde foramen incisivo a espina nasal posterior), Tipo II (foramen incisivo a la sutura palatina transversal), Tipo III (zona posterior del maxilar a la parte más posterior de la espina nasal) y tipo IV (solo zona posterior maxilar). Al Quran y AlDwairi (10) clasifican los TP según características clínicas en torus planos, lobulares, nodulares o en forma de huso. Otra clasificación que sugiere Martins y cols. (7) es según número: unilateral único, unilateral múltiple, bilateral único o bilateral múltiple.

La etiología del torus se relaciona genéticamente como herencia a un mismo gen autosómico dominante (11), ligada al cromosoma Y, excluyéndose la herencia ligada al cromosoma X $(10,12)$, sugiriendo penetrancia incompleta alrededor del 9,1\% (12). También se mencionan factores ambientales desencadenantes como el estrés, hiperfunción masticatoria, abrasión dentaria o bruxismo (13), enfermedades periodontales y procesos infecciosos del tejido conectivo cercano a la línea gingival (14). Otros autores mencionan hábitos alimenticios relacionados con el nivel de consumo de ácidos grasos polisaturados y vitamina D, involucrados en el proceso de crecimiento óseo (15), así como fuerzas musculares ejercidas en la región (7).

En cuanto a su epidemiología, el torus ha reportado tener una asociación positiva entre la edad, frecuencia de ocurrencia y tamaño (13), siendo la forma única más prevalente que la múltiple, mayor en edades tempranas. Generalmente, predomina la forma simétrica, y cuando es unilateral, se encuentra con mayor frecuencia en el lado derecho (11), en la zona del canino (40,5\%), seguida la zona premolar $(25,7 \%)$ (8). En habitantes de Norte América el torus alcanza una prevalencia del $63,9 \%$, en individuos con grandes músculos masticadores (10). Cabe destacar que tanto el TM como el TP se encuentran asociados entre sí en un 3\% a 8\% de los casos (10). Estas dos variaciones anatómicas son relativamente comunes y afectan aproximadamente entre el $10 \%$ (13) al $30 \%(16)$ de la población mundial.

Según etnias, se estima que entre un $20 \%$ a $25 \%$ de la población Asiática, nativa Indígena Americana y Esquimal presentan torus (7), con mayor prevalencia en hombres que en mujeres en proporción 4:1 (14), entre los 35 y 65 años (16). En países como Tailandia, la prevalencia de torus es de 23,1\% (17) y Turquía un 30,9\% (18); en cambio en América del Sur se ha reportado una baja prevalencia para esta condición (10).

El objetivo de este estudio fue determinar la prevalencia de torus en población Chilena-específica de Temuco, IX región, y relacionar esta condición anatómica con algunos factores asociados.

\section{MATERIAL Y MÉTODOS}

Se realizó un estudio transversal, descriptivo, observacional. Fueron seleccionados al azar 159 pacientes de un total de 678 inscritos entre el periodo mayoagosto del año 2010 en las Clínicas Odontológicas de la Universidad de la Frontera, Temuco. Se les informó acerca del estudio y se solicitó su consentimiento para realizar la evaluación. Se realizó una inspección visual de la cavidad oral, con un espejo de examen, a través del cual se observó específicamente el paladar y reborde interno lingual mandibular para verificar la presencia de exostosis en dichas zonas. En casos de pacientes portadores de aparatos protésicos, fueron retirados previo al examen.

Para confirmar la existencia de torus, se realizó palpación intraoral con el dedo índice. Se consideró como torus a toda elevación sólida, dura y circunscrita. Una vez realizado el diagnóstico se completó una ficha recabando los siguientes datos:

- Sexo

- Edad: considerada según mayor o menor de 30 años.

- Etnia: considerado según Mapuche o no Mapuche.

- Fumador 
- Onicofagia

- Número de piezas dentarias: considera edéntulo total, parcial o dentado total.

- Zona desdentada: Considera zona superior, inferior o ambas.

- Presencia de prótesis.

- Ubicación del torus: Mandibular o palatino.

- Características clínicas del torus: Mandibular bilateral o unilateral y palatino.

A partir de las edades de los pacientes, se calculó una mediana de 30 años separando la muestra dos grupos, menores o igual a 30 años y mayores de 30 años. La etnia fue determinada mediante los apellidos. Se consideró indígena perteneciente a la etnia mapuche a aquellos sujetos que tuviesen al menos uno de sus apellidos y de sus padres de origen mapuche, y como no indígena a aquellos que tienen sólo apellidos de origen chileno-español. La onicofagia se determinó según lo relatado por el paciente. Se consideró como fumador quienes habían consumido al menos un cigarrillo diariamente.

Los pacientes con presencia de torus se sometieron a la toma de fotografía intraoral de la zona comprometida con una cámara digital Canon PowerShot A430.

Al finalizar la recolección de datos estos fueron tabulados en una tabla Excel para ser posteriormente analizados con el software STATA v. 9.0. trabajando con un valor se significación de un $5 \%$.

\section{RESULTADOS}

En relación al sexo, del total de la muestra 99 correspondieron a mujeres $(62,26 \%)$ y $60(37,74 \%)$ a hom- bres. La prevalencia total de torus fue de 5,66\%, y al analizarla según sexo, esta fue de $6,93 \%$ en mujeres $y$ de $3,45 \%$ en hombres con un valor $p=0,3603$.

Según la edad, la prevalencia de torus en mayores de 30 años fue de $6,33 \%$, mientras que en pacientes menores o igual a 30 años fue de un $5 \%(p=0,7109)$. La prevalencia de torus en pacientes de etnia Mapuche fue de $14,28 \%$ y $4,34 \%$ para los no Mapuches $(p=0,064)$.

De acuerdo a su localización en el total de la muestra, el $77,78 \%$ de los torus tuvo una localización mandibular (lingual) y un 22,22\% palatino. Un $11,11 \%$ correspondían a torus de tipo mandibular unilateral y el $66,67 \%$ bilateral, mientras que el $22,22 \%$ fueron torus palatinos ubicados en el plano mediano (valor $p=0,0427$ ) (Figura 1 A, B y C).

$\mathrm{Al}$ analizar los factores asociados y la presencia de torus, se encontró que un 6,38\% correspondieron a pacientes fumadores, mientras que la onicofagia estuvo presente tan solo en el 1,82\% de la muestra. Ninguna de estos asociaciones mostró valores significativos $(p=0,7984)$.

Del total de la muestra el $64,78 \%$ fueron pacientes parcialmente dentados, en tanto el 35,22\% restante resultaron ser pacientes con dentición completa. No se reportaron casos de edentulismo total. De los pacientes parcialmente dentados un $23,36 \%$ fueron maxilares y 20,56\% mandibulares. La ausencia de una o más piezas dentales en el maxilar o la mandíbula se observó en el 56,07\%. Al asociar esta variable con la presencia de torus, estos se observaron en el 6,8\% de los pacientes parcialmente desdentados y en el $3,57 \%$ de los dentados. Según la zona des-

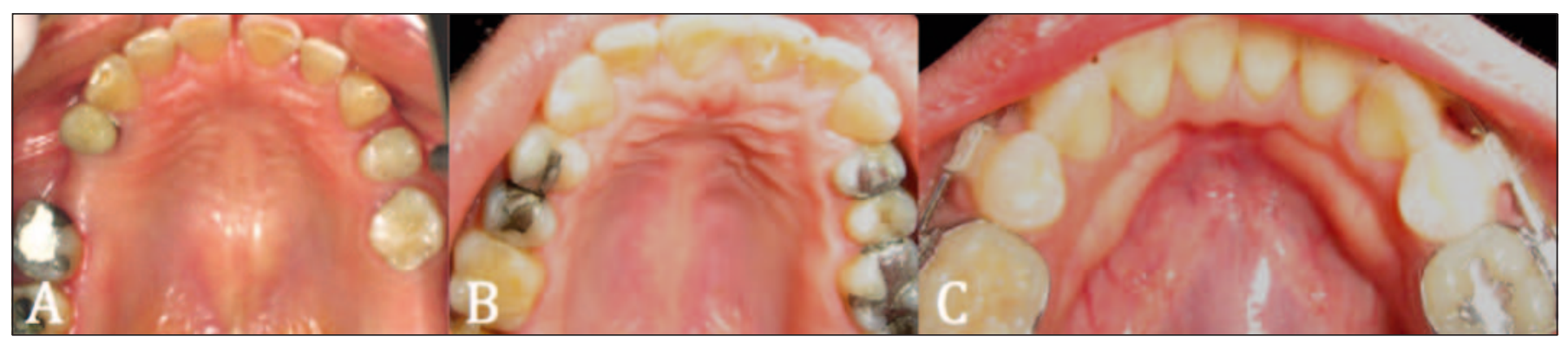

Fig. 1. A) Torus palatino, paciente sexo femenino, $>30$ años. B) Torus palatino, paciente sexo masculino < 30 años. C) Torus mandibular bilateral, paciente sexo femenino, $<30$ años. 
dentada, el torus estuvo presente en el $12 \%$ de los pacientes parcialmente desdentados maxilares y en el 6,67\% de los parcialmente desdentados mixtos (maxilar y mandibular).

Los pacientes portadores de prótesis correspondieron al $20,25 \%$ de la muestra, de los cuales $77,42 \%$ portaba prótesis superior, $6,45 \%$ prótesis inferior y $16,13 \%$ ambas arcadas. Al asociar esta condición a la presencia de torus, en el 9,37\% de los pacientes portadores de prótesis se observó algún tipo de torus, mientras que sólo un 4,8\% de los pacientes con torus no fue portador de prótesis.

En la tabla 1 se pueden observar en detalles la asociación de las diferentes variables con los torus.

\section{DISCUSIÓN}

Las exostosis orales o torus constituyen una variación anatómica de importancia en la odontología. Sin embargo, los estudios en Chile acerca de su frecuencia es escasa.

La prevalencia de torus encontrada en este estudio fue de un $5,66 \%$, coincidiendo con la baja prevalencia reportada en América del Sur (10). Esta cifra es menor que lo señalado en el resto del mundo por autores como Gorsky y cols. (21\%) (12), Bruce y cols. (14\%) (19), Al-Bayaty y cols. (12,3\%) (20) y Meza (10\%) (11). La prevalencia según sexo encontrada en este estudio fue de $6,93 \%$ en mujeres y $3,45 \%$ en hombres, sin ser estadísticamente significativa. Esto coincide con lo descrito por Ponzoni y cols. (4), García y cols. (21) y Jainkittivong $\&$ Langlais (8) quienes manifiestan un leve predominio de esta condición en el sexo femenino. Sin embargo, Pechenkina $E$ Benfer (13) se contraponen a estos resultados, reportando los torus más prevalentes en hombres $(80 \%)$ que en mujeres (20\%) de Sudáfrica; situación igualmente descrita por Martins y cols. (7).

La presencia de torus se encontró en el 6,33\% de los mayores de 30 años y en el $5 \%$ en menores o iguales de 30 años, sin observar diferencias significativas, ya que la mediana de 30 años de edad no demuestra que el torus tenga relación directa con la edad. Según Raldi y cols. (16) la mayor frecuencia se en-
TABLA 1.- PREVALENCIA DE TORUS SEGÚN LAS VARIABLES ESTUDIADAS EN UINA MUESTRA POBLACIÓN-ESPECÍFICA DE TEMUCO, CHILE

\begin{tabular}{|c|c|c|c|}
\hline Variables & Prevalencia & $\mathbf{n}$ & Valor $\mathrm{p}$ \\
\hline $\begin{array}{l}\text { Ubicación } \\
\text { - Mandibular } \\
\text { - Palatino }\end{array}$ & $\begin{array}{l}22,00 \% \\
78,00 \% \\
\end{array}$ & $\begin{array}{l}2 / 9 \\
7 / 9\end{array}$ & - \\
\hline $\begin{array}{l}\text { Caract. clínicas } \\
\text { - TM unilateral } \\
\text { - TM bilateral } \\
\text { - TP }\end{array}$ & $\begin{array}{l}11,11 \% \\
66,67 \% \\
22,22 \%\end{array}$ & $\begin{array}{l}1 / 9 \\
6 / 9 \\
2 / 9\end{array}$ & $0,0427 *$ \\
\hline $\begin{array}{l}\text { Sexo } \\
\text { - Femenino } \\
\text { - Masculino }\end{array}$ & $\begin{array}{l}6,93 \% \\
3,45 \%\end{array}$ & $\begin{array}{l}7 / 101 \\
2 / 58\end{array}$ & 0,3603 \\
\hline $\begin{array}{l}\text { Edad } \\
\cdot \leq 30 \\
\cdot>30\end{array}$ & $\begin{array}{l}5,00 \% \\
6,33 \%\end{array}$ & $\begin{array}{l}4 / 80 \\
5 / 79\end{array}$ & 0,7169 \\
\hline $\begin{array}{l}\text { Etnia } \\
\text { - Mapuche } \\
\text { - No mapuche }\end{array}$ & $\begin{array}{r}14,28 \% \\
4,34 \%\end{array}$ & $\begin{array}{l}3 / 21 \\
6 / 138\end{array}$ & 0,064 \\
\hline $\begin{array}{l}\text { Factor asociado } \\
\text { - Onicofagia } \\
\text { - Sin onicofagia } \\
\text { - Fumador } \\
\text { - No fumador }\end{array}$ & $\begin{array}{l}1,82 \% \\
7,69 \% \\
6,38 \% \\
5,36 \%\end{array}$ & $\begin{array}{l}1 / 55 \\
8 / 104 \\
3 / 47 \\
6 / 112\end{array}$ & 0,7984 \\
\hline $\begin{array}{l}\text { Dentición } \\
\text { - Total } \\
\text { - Parcial }\end{array}$ & $\begin{array}{l}3,57 \% \\
6,80 \%\end{array}$ & $\begin{array}{l}2 / 56 \\
7 / 103\end{array}$ & 0,4006 \\
\hline $\begin{array}{l}\text { Zona desdentada } \\
\text { - Superior } \\
\text { - Superior e inferior }\end{array}$ & $\begin{array}{r}12,00 \% \\
6,67 \%\end{array}$ & $\begin{array}{l}3 / 25 \\
4 / 60\end{array}$ & 0,2516 \\
\hline $\begin{array}{l}\text { Prótesis } \\
\text { - Con prótesis } \\
\text { - Sin prótesis }\end{array}$ & $\begin{array}{l}9,37 \% \\
4,80 \%\end{array}$ & $\begin{array}{l}3 / 32 \\
6 / 127\end{array}$ & 0,3147 \\
\hline
\end{tabular}

${ }_{p}^{*}<0,05$, diferencia estadisticamente significativa. 
cuentra en pacientes entre 35-65 años, lo que es coincidente con los resultados expuestos en este estudio y con Pechenkina $E$ Benfer (13), Eggen y Natvig (22) y Haugen (23), quienes reportan que la presencia de torus aumentan con la edad, presentándose en $31,3 \%$ cercano a los 30 años, hasta un $57,9 \%$ en mayores de 50 años. Por otra parte, Meza (11) reporta que la mayoría de los torus son encontrados entre los 11 a 30 años. Por otro lado, Eroğlu y Erdal (15) y Al-Bayaty y cols. (20) manifiestan que raramente esta condición se ve en niños y su crecimiento comienza después de los 10 años de edad, aumentando su frecuencia con la edad al comparar grupos jóvenes y de edad avanzada.

La relación encontrada entre la presencia de torus y etnia Mapuche sugiere que existe una tendencia mayor a encontrar exostosis en etnia originarias. Según Martins y cols. (7) existe una asociación positiva entre algunas etnias y la presencia de torus, destacando que entre un $20 \%$ a $25 \%$ de la población de Asiáticos nativos, Indígenas Americanos y Esquimales presentan esta condición. Algunos autores como Meza (11), García y cols. (21) y Gorsky y cols. (12) coinciden que existe una mayor prevalencia de exostosis en algunos grupos raciales más que en otros.

Con respecto los factores asociados a esta condición, en los pacientes fumadores se observó una mayor prevalencia de torus, pero sin diferencias estadísticamente significativas. Meza (11) explica que factores como medicación, índice de masa corporal, fumar y otros que afectan la densidad de ósea, no disminuyen, ni tienen una relación directa con el tamaño ni con la aparición de los torus. Por otra parte, la onicofagia no tuvo relación significativa con la presencia de torus, ya que la mayor prevalencia se observó en pacientes que no presentaban el hábito de morderse las uñas.

Se observaron exostosis orales en el 6,8\% del los pacientes parcialmente dentados y en el 3,57\% de los dentados totales. De los pacientes parcialmente dentados con torus, el 42,85 \% correspondía a pacientes desdentados parciales maxilares, siendo uno de estos portador de prótesis superior. Además, el $57,1 \%$ eran pacientes con torus y desdentados tanto superior como inferior, de los cuales, solo 2 pacien- tes portaban prótesis en ambas arcadas. La relación posible y la mayor tendencia observada en los pacientes parcialmente dentados podría relacionarse con lo planteado por Martins y cols. (7), quienes proponen como patogénesis de la aposición ósea al aumento de fuerzas musculares ejercidas en la región debido a la ausencia de piezas dentarias. Es importante destacar que la asociación entre los torus y los pacientes parcialmente desdentados podría tener un sesgo debido a que los profesionales se percatan del problema al momento de realizar o asentar las prótesis por no realizar un examen acabado de todos los pacientes.

El TM se presentó con mayor frecuencia $(77,78 \%)$ en relación al TP (22,22\%). Los resultados obtenidos concuerdan con lo planteado por $\mathrm{Al}$ Quran y $\mathrm{Al}$ Dwairi (10) donde la prevalencia de TP fue de $29,8 \%$, mientras que la de TM fue significativamente superior (42,6\%). Gorsky y cols. (12) confirman que existe mayor prevalencia de TM. En países como Tailandia, la prevalencia del TP fue 23,1\% (17), en Turquía 30,9\% (18), a diferencia de lo evidenciado en América del Sur, donde la prevalencia es baja (10), coincidiendo con nuestros resultados. El torus de tipo bilateral predominó con localización mandibular $(66,67 \%)$, seguido del TP en la línea mediana $(22,22 \%)$, mientras que el unilateral mandibular mostró el menor porcentaje $(11,11 \%)$. Estos resultados fueron los únicos en los que se observaron cifras estadísticamente significativas. Nuestros resultados contrastan con lo planteado por Pechenkina y Benfer (13) quienes manifiestan que los torus unilaterales son más prevalentes que los bilaterales.

La presencia de torus suponen un desafío en la restauración principalmente de arcos edéntulos. Su tratamiento está indicado en aquellos casos que presenten gran tamaño, alteren la función, oclusión, produzcan trauma de la superficie como ulceración, ya que la tener una delgada mucosa que los reviste puede ulcerarse secundariamente a un trauma, causado por ejemplo con alimentos duros o las presiones normales de una base protésica, o en casos que interfieran en la fisiología de la fonación, masticación, dicción, deglución o posición normal de la lengua (2).

En caso de requerir tratamiento, éste apunta principalmente a la eliminación quirúrgica mediante su 
tallado o remoción, cortándose desde la base de la unión (13), ya que en muchas ocasiones los torus evitan la creación de un buen sello protésico (24). Cuando son asintomáticos o de un tamaño pequeño, la literatura plantea que no es necesaria su remoción, si no que al momento de construir prótesis esta se puede modificar de manera adecuada (25). Por otra parte, en casos específicos, se evita su remoción ya que estas elevaciones óseas podrían ser utilizadas como sitios de recolección de hueso cortical autólogo en procedimientos quirúrgicos para reemplazar tejido óseo perdido (12).

\section{CONCLUSIONES}

Por todo lo anteriormente expuesto podemos concluir que existe una prevalencia de exostosis o torus concordante a la media de América del Sur, con un predominio en el sexo femenino. El TM fue más frecuente en su forma bilateral, seguido por el TP. Además se observó una tendencia mayor de torus en la etnia Mapuche y en pacientes mayores de 30 años. El tabaco y la onicofagia se descartaron como posibles factores asociados con la presencia de torus. Finalmente, existió una correlación positiva entre la ausencia de piezas dentarias y la presencia de torus.

\section{BIBLIOGRAFÍA}

1. Lee SP, Paik KS, Kim MK. Variations of the prominences of the bony palate and their relationship to complete dentures in Korean skulls. Clin Anat 2001;14:324-9.

2. Neville BW, Damm DD, Allen CM, Bouquot JE. Oral and Maxillofacial Pathology. 2nd ed. Philadelphia: W.B. Saunders, 2002.

3. Sisman Y, Ertas ET, Gokce C, Akgunlu F. Prevalence of torus palatinus in cappadocia region population of Turkey. Eur J Dent 2008;2: 269-75.

4. Ponzoni D, Marques J, Pirani A, Machado de Souza R, Frias, R. Remoção cirúrgica de toro palatino para confecção de prótese total con- vencional - indicações de diferentes incisões. RFO UPF 2008; 13:66-70.

5. Belsky JL, Hamer JS, Hubert JE, Insogna K, Johns W. Torus palatinus: a new anatomical correlation with bone density in postmenopausal women. J Clin Endocrinol Metab 2003;88: 2081-6.

6. Cagirankaya LB, Kansu O, Hatipoglu MG. Is torus palatinus a feature of a well-developed maxilla? Clin Anat 2004;17:623-5.

7. Martins MD, Lata SP, Martins MAT, Bussadori SK, Fernandes KPS. Toro Palatino e Mandibular: revisão de literatura. ConScientiae Saúde 2007;6:57-62.

8. Jainkittivong A, Langlais RP. Buccal and palatal exostoses: prevalence and concurrence with tori. Oral Surg Oral Med Oral Pathol Oral Radiol Endod 2000;90:48-53.

9. Bernal Balaez A, Moreira Diaz E, Rodriguez Perez I. Prevalence of torus palatinus and torus mandibularis in the city of Havana. Rev Cubana Estomatol 1983;20:126-31.

10. Al Quran FA, Al-Dwairi ZN. Torus palatinus and torus mandibularis in edentulous patients. $\mathrm{J}$ Contemp Dent Pract 2006;7:112-9.

11. Meza JL. Cavidad Oral: Torus palatinus y Torus mandibularis. Rev. Gastroenterol. Perú 2004; 24:343-8.

12. Gorsky M, Bukai A, Shohat M. Genetic influence on the prevalence of torus palatinus. Am J Med Genet 1998;75:138-40.

13. Pechenkina EA, Benfer RA Jr. The role of occlusal stress and gingival infection in the formation of exostoses on mandible and maxilla from Neolithic China. Homo 2002;53:112-30.

14. Ihunwo AO, Phukubye P. The frequency and anatomical features of torus mandibularis in a Black South African population. Homo 2006; 57:253-62. 
15. Eroğlu S, Erdal YS. Why did the frequency of palatine torus increase in the ancient Anatolian populations? Homo 2008;59:365-82.

16. Raldi FV, Nascimento RD, Sá-Lima JR, Tsuda CA, Moraes MB. Excision of an atypical case of palatal bone exostosis: a case report. J Oral Sci 2008; 50:229-31.

17. Reichart PA, Neuhaus F, Sookasem M. Prevalence of torus palatinus and torus mandibularis in Germans and Thai. Community Dent Oral Epidemiol 1988;16:61-4.

18. Yildiz E, Deniz M, Ceyhan O. Prevalence of torus palatinus in Turkish schoolchildren. Surg Radiol Anat 2005;27:368-71.

19. Bruce I, Ndanu TA, Addo ME. Epidemiological aspects of oral tori in a Ghanaian community. Int Dent J 2004;54:78-82.

20. Al-Bayaty HF, Murti PR, Matthews R, Gupta PC. An epidemiological study of tori among 667 dental outpatients in Trinidad $E$ Tobago, West Indies. Int Dent J 2001;51:300-4.

21. García-García AS, Martínez-González JM, GómezFont R, Soto-Rivadeneira A, Oviedo-Roldán L. Current status of the torus palatinus and torus mandibularis. Med Oral Patol Oral Cir Bucal 2010;15:e353-60.

22. Eggen S, Natvig B. Concurrence of torus mandibularis and torus palatinus. Scand J Dent Res 1994;102:60-3.

23. Haugen LK. Palatine and mandibular tori. A morphologic study in the current Norwegian population. Acta Odontol Scand 1992;50:65-77.

24. Abrams S, Hellen W. Fabrication of an overdenture covering a torus palatinus using a combination of denture base materials: a case report. Dent Today 2006;25:74-7.

25. Shah DS, Sanghavi SJ, Chawda JD, Shah RM. Prevalence of torus palatinus and torus mandibularis in 1000 patients. Indian J Dent Res 1992;3:107-10.

\section{CORRESPONDENCIA}

Ramón Fuentes Fernández

Depto. Odontología Integral

Facultad de Medicina

Universidad de La Frontera

Manuel Montt 112, Temuco, Chile.

E-mail: rfuentes@ufro.cl 